\title{
Food Addiction in Bulimia Nervosa: Clinical Correlates and Association with Response to a Brief Psychoeducational Intervention
}

Inés Hilker ${ }^{1,9}$; Isabel Sánchez ${ }^{1}$; Trevor Steward ${ }^{1,2}$; Susana Jiménez-Murcia ${ }^{1,2,3}$, Roser Granero $^{2,4}$; Ashley N. Gearhardt ${ }^{5}$; Rita Cristina Rodríguez-Muñoz ${ }^{1}$; Carlos Dieguez ${ }^{2,6}$; Ana B. Crujeiras ${ }^{2,7}$; Iris Tolosa-Sola ${ }^{1}$; Felipe F. Casanueva ${ }^{2,7}$; José M Menchón ${ }^{1,3,8}$; Fernando Fernández-Aranda ${ }^{1,2,3 *}$

\section{$\underline{\text { Affiliations }}$}

${ }^{1}$ Department of Psychiatry, University Hospital of Bellvitge-IDIBELL, Barcelona, Spain.

${ }^{2}$ Ciber Fisiopatologia Obesidad y Nutrición (CIBERObn), Instituto Salud Carlos III, Barcelona, Spain

${ }^{3}$ Department of Clinical Sciences, School of Medicine, University of Barcelona, Spain

${ }^{4}$ Department of Psychobiology and Methodology. University Autònoma of Barcelona, Spain.

${ }^{5}$ Department of Psychology, University of Michigan, Ann Arbor, MI, USA

${ }^{6}$ Department of Physiology, CIMUS, University of Santiago de Compostela-Instituto de Investigación Sanitaria, Santiago de Compostela, Spain

${ }^{7}$ Laboratory of Molecular and Cellular Endocrinology, Research Area, Complejo Hospitalario Universitario de Santiago de Compostela, A Coruña, Spain

${ }^{8}$ Ciber Salud Mental (CIBERSAM), Instituto Salud Carlos III, Barcelona, Spain

${ }^{9}$ Hospital Clinic of Barcelona, Barcelona, Spain

\section{*Correspondence to:}

Fernando Fernández-Aranda, Ph.D., Head of Eating Disorders Unit.

Department of Psychiatry. Bellvitge University Hospital-IDIBELL and CIBERobn.

c/ Feixa Llarga s/n, 08907 L'Hospitalet de Llobregat (Barcelona, Spain).

Tel. +34 9326079 88; Fax. +349326076 58 .

E-mail: ffernandez@bellvitgehospital.cat

This is the author manuscript accepted for publication and has undergone full peer review but has not been through the copyediting, typesetting, pagination and proofreading process, which may lead to differences between this version and the Version of Record. Please cite this article as doi: $10.1002 /$ erv.2473

This article is protected by copyright. All rights reserved. 


\begin{abstract}
Food addiction (FA) has been examined in different populations. Although high FA levels are associated with greater eating-disorder severity, few studies have addressed how FA relates to treatment outcome. Goals: (1) to determine whether a brief intervention for bulimia nervosa $(\mathrm{BN})$ reduces $\mathrm{FA}$ diagnosis or severity compared to baseline; and (2) to determine if FA is predictive of treatment outcome. Method: 66 female BN patients participated in the study. The Yale Food Addiction Scale was administered at two time points: prior to- and following a 6-week intervention. The number of weekly binging/purging episodes, dropout and abstinence from bulimic behavior were used as primary outcome measures. Results: This brief intervention reduced FA severity and FA diagnosis in the 55 patients who completed treatment. FA severity was a short-term predictor of abstinence from binging/purging episodes after treatment $(p=.018)$. Conclusions: FA appears to be prevalent in BN although FA severity can improve following a short-term intervention.
\end{abstract}

\title{
Keywords:
}

bulimia nervosa; food addiction; treatment; outcome; clinical

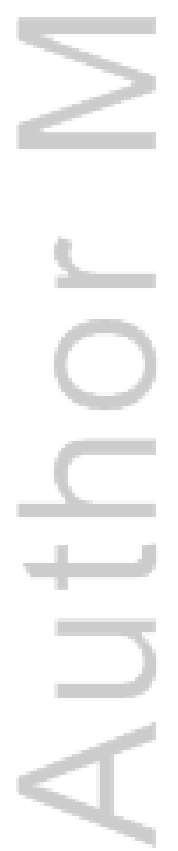




\section{Introduction}

Since the development of the Yale Food Addiction Scale (YFAS) (Gearhardt, Corbin, \& Brownell, 2009), the first validated tool for the measurement of food-related addictive behaviors, research in food addiction (FA) has steadily grown. The FA construct upholds that obesity and eating disorders (ED) can be driven by an addictive response to certain types of foods (e.g., high sugar, high fat) in a manner that is akin to substances of abuse. As such, the YFAS is based upon symptomatic criteria for substance dependence as defined by the DSM-IV-TR. Nevertheless, food addiction (FA) remains a controversial topic and the question of whether FA is more similar to a substance use disorder or is better classified as a behavioral addiction is still unresolved (Hebebrand et al., 2014).

To date, the prevalence and correlates of FA diagnosis have been examined in a range of different populations. Cross-sectional studies have explored FA prevalence in nonclinical populations (Gearhardt et al., 2009), abnormal weight and eating populations, namely in people with obesity (Meule, Hermann, \& Kübler, 2015), and in individuals with eating disorders, namely binge eating disorder (Gearhardt et al., 2012; Gearhardt, White, Masheb, \& Grilo, 2013) and bulimia nervosa (BN), along with other ED subtypes (Granero et al., 2014; Meule \& Gearhardt, 2014a; Pursey, Stanwell, Gearhardt, Collins, \& Burrows, 2014).

FA symptomatology is associated with high levels of negative affect and depression, high general psychopathology, eating pathology and a high body mass index (Gearhardt, Boswell, \& White, 2014; Gearhardt, White, Masheb, \& Grilo, 2013; Granero, Hilker, Agüera, Jiménez-Murcia, et al., 2014). Moreover, high scores in FA are linked to dieting, an earlier age of excess-weight onset and binge eating frequency (Gearhardt et al., 2013). Personality traits also play a role in being vulnerable to develop FA; ED patients with FA have been found to report lower self-directedness, higher negative urgency and a greater lack of perseverance (Wolz et al., 2016).

This article is protected by copyright. All rights reserved. 
A limited number of studies have addressed the question of how FA is related to treatment outcomes in weight-loss seeking populations, and have provided contradictory results (Burmeister, Hinman, Koball, Hoffmann, \& Carels, 2013; Clark \& Saules, 2013; Lent, Eichen, Goldbacher, Wadden, \& Foster, 2014; Pepino, Stein, Eagon, \& Klein, 2014). Whereas FA symptoms were negatively correlated with weight loss in some studies (Burmeister et al., 2013; Clark \& Saules, 2013), others found that neither FA diagnosis nor the number of FA symptoms were associated with successful weight loss (Lent et al., 2014). However, another study showed that bariatric surgeryinduced weight loss led to FA remission and improved several eating behaviors associated with FA (Pepino et al., 2014). Another recent study explored the stability of FA diagnosis and FA symptomatology in a non-clinical population over 18 months. The study concluded that the number of FA symptoms and FA diagnosis remained relatively stable over time (Pursey, Collins, Stanwell, \& Burrows, 2016). The authors also tested if engaging in weight loss behaviors could explain within person changes, but did not find a significant correlation. This observed stability of FA symptoms suggests that the YFAS could be an appropriate tool for assessing addictive-like eating behaviors prior to- and following clinical interventions.

To our knowledge, no study has yet to explore whether ED treatment reduces FA symptomatology. This study presents the first longitudinal examination of FA and ED treatment response. It is worth noting however that Meule and colleagues explored the differences in FA prevalence between current $\mathrm{BN}$ patients and recovered $\mathrm{BN}$ patients in a cross-sectional study (Meule, von Rezori, \& Blechert, 2014). This study found that FA diagnosis and symptomatology were significantly lower in recovered BN patients than in currently ill patients.

The objectives of the present study were twofold: (1) to determine if brief psychoeducational treatment for $\mathrm{BN}$ has an impact on FA diagnosis and on reducing FA symptomatology compared to baseline levels; and (2) to determine if FA has a predictive capacity in terms of short-term treatment outcome in BN. For this purpose, FA and other clinical variables (namely the number of binge and purging episodes, 
eating symptomatology and general psychopathology) were assessed prior to- and following a brief psychoeducational intervention for BN.

In line with previous BN studies (Granero et al., 2014; Meule \& Gearhardt, 2014a; Pursey et al., 2014), we expected BN patients to present a high prevalence of FA diagnosis. We also expected FA severity to positively correlate with eating symptomatology and general psychopathology. Moreover, given that brief psychoeducational treatment has proved to be effective as a first stage approach to reduce binging and purging behaviors (Davis, Olmsted, \& Rockert, 1990; FernándezAranda et al., 2009), and that recovered BN patients present less FA symptoms (Meule et al., 2014), we expected FA severity levels to significantly reduce following treatment. Lastly, we also hypothesized that higher FA severity at baseline would be associated with worse treatment outcomes.

\section{Methods}

\section{Participants and procedure}

All participants in this sample were women with $\mathrm{BN}$ who were consecutively referred to the Eating Disorders Unit at Bellvitge University Hospital between September 2013 and December 2015 ( $n=66$ ). Patients were diagnosed according to DSM-5 criteria (APA, 2013) by means of a semi-structured, face-to-face interview conducted by $\mathrm{PhD}$-level psychologists. Final diagnoses were made by the Unit psychologists following the completion of the study questionnaires. Inclusion criteria to the study were: 1) being female; 2) fulfilling DSM-5 criteria for BN; 3) being between 18 to 65 years old.

Questionnaires were administered at two time points: before and upon completion of psychoeducational treatment. Additional information concerning clinical variables was also taken during the completion of the questionnaires by means of an interview conducted by a member of the Unit staff.

During the weeks prior to admission to treatment and throughout the entirety of the program, patients recorded their eating behavior and the frequency of binge eating and 
purging episodes by means of a food diary (Fernández-Aranda \& Turon, 1998) that was shared with their therapist on a weekly basis.

The present study was carried out in accordance with the latest version of the Declaration of Helsinki. The Bellvitge University Hospital Ethics Committee approved this study, and written informed consent was obtained from all participants.

\section{Assessment}

The Yale Food Addiction Scale (YFAS; Gearhardt et al., 2009)

The YFAS is a 25-item self-report instrument, based upon the seven symptomatic criteria for substance dependence set out in the DSM-IV-TR (Gearhardt et al., 2009) (see Table 2). It includes two additional items that assess significant clinical impairment or distress because of overeating. When at least three criteria and significant clinical impairment are reported, a diagnosis of FA is given. Moreover, a symptom count, indicating FA severity, can be measured to provide a score between 1 and 7 .

The YFAS requests that respondents answer questions according to their eating behavior in the past 12 months. For the assessment upon completing psychoeducational treatment, patients were instructed to complete this questionnaire keeping in mind their present eating behavior. The Spanish version of the YFAS has been validated and have shown good psychometrical properties (Granero et al., 2014).

The Symptom Checklist Revised (SCL-90-R; Derogatis, 1990)

The Symptom Checklist Revised (SCL-90-R) is a 90-item questionnaire that is widely used for the measurement of self-reported overall psychological distress and psychopathology (Derogatis, 1990). It is scored using the following dimensions: somatization, obsessive-compulsive, interpersonal sensitivity, depression, anxiety, hostility, phobic anxiety, paranoid ideation and psychoticism. The Spanish validation of this instrument has shown a mean internal consistency of $\alpha=0.75$ (Ignacio \& Sánchez, 2002; Martínez-Azumendi, Fernández-Gómez, \& Beitia-Fernández, 2001). 
Eating Disorders Inventory-2 (EDI-2; Garner, 1991)

Eating Disorders Inventory-2 (EDI-2) is a 91-item self-report questionnaire that assesses the following features of eating disorders: drive for thinness, bulimia, body dissatisfaction, ineffectiveness, perfectionism, interpersonal distrust, interoceptive awareness, maturity fears, asceticism, impulse regulation and social insecurity (Garner, 1991). A Spanish version of this questionnaire has been validated (Garner, 1998).

\section{Treatment protocol}

This outpatient treatment program consisted of a brief group psychoeducational intervention based on Davis, Olmsted, \& Rockert's (1990) model. This model is designed to promote symptom management and each meeting is structured around specific themes such as "the multidetermined and self-perpetuating nature of $\mathrm{BN}$ " and "the nondieting approach to eating". The intervention included six weekly outpatient sessions lasting $90 \mathrm{~min}$ each. The treatment protocol was explained to the group members and therapeutic materials were provided during the initial sessions. The main aims of this intervention are to offer educational information about $\mathrm{BN}$, to show patients the importance of assuming an active role in the resolution of their disorder and to encourage them to establish a pattern of regular and healthy eating, as well as to reduce dieting. Additionally, information from food diaries was used as a therapeutic tool during the sessions. For example, information from these diaries might be discussed with the therapist and the rest of group members in order to increase awareness about bulimic symptoms. Nonetheless, given the main aims of this intervention, delving into individual patient problems was avoided during sessions.

This treatment has been shown to be effective in significantly reducing eating psychopathology and approximately 30\% of treated patients do not present either binging or purging behavior at the end of the treatment (Fernández-Aranda et al., 2009; Wilson, Fairburn, \& Agras, 1997). A total of eleven consecutive psychoeducational groups, with a range of 6-8 patients per group, made up the study sample. 


\section{Outcome measures}

The number of binging/purging episodes per week, based on entries from a food diary, was used as the primary outcome measure to assess response to treatment. Participants were categorized (at baseline and after treatment) into five groups based on DSM-5 severity criterion for $\mathrm{BN}$ : null (0 binging/purging episodes per week), mild (1-3 episodes), moderate (4-7 episodes), severe (8-13 episodes) and extreme (14 or more episodes) (APA, 2013). Patients who completed treatment were re-classified into two categories depending on the presence, or absence, of a reduction in severity: "goodresponders" (change to a lower severity group after treatment, i.e. from severe to moderate, mild or null, or from moderate to mild or null) versus "poor-responders" (failing to reduce severity group after treatment). Dropout from treatment and achieving complete abstinence from binging/purging episodes during the final week of treatment were also used as primary treatment outcomes. As secondary outcome measures, we compared pre- and post-treatment scores for ED symptomatology, by means of the EDI2, and general psychopathology, by means of the SCL-90-R.

\section{Statistical analysis}

Statistical analysis was carried out with SPSS 21. First, independent t-tests compared the mean number of YFAS symptoms between "good" and "poor" responders. The effect size for mean differences was estimated through the $95 \%$ confidence interval (95\% CI) for mean differences and Cohen's- $d$ coefficient $(|d|>0.50$ was considered moderate effect size and $|d|>0.80$ was considered high effect size). Second, binary logistic regressions explored the predictive capacity of FA severity at baseline on our three primary outcomes: good response to treatment, extinction of binging/purging episodes and dropout. Global predictive capacity for the logistics was estimated by the Nagelkerke's pseudo- $\mathrm{R}^{2}$ coefficient, and discriminative ability by the area under the ROC curve (AUC).

Additionally, paired t-tests compared pre-post changes for quantitative ED measures (number of binges and purges per week, EDI-2 scores, SCL-90-R scores and FA 
severity) and McNemar tests compared the pre-post changes for each FA symptom (presence versus absence) and for FA diagnosis (positive versus negative diagnosis).

In this study, increases in Type-I error due to multiple statistical comparisons were controlled via Finner's method (Finner, 1993), a procedure in Family-wise error rate stepwise systems that offers more powerful tests than classical Bonferroni-correction (Finner, 1993).

\section{Results}

Study sample

The mean age for the $N=66$ participants who began treatment was 29.2 years ( $\mathrm{SD}=9.2$ ), the mean age of onset for $\mathrm{BN}$ was 18.6 years $(\mathrm{SD}=5.6)$ and the mean duration of the disorder was 10.6 years $(\mathrm{SD}=9.1) .42 .5 \%$ of patients reported between 1 to 3 previous ED treatment attempts (the remaining $57.5 \%$ had not previously sought help for eatingrelated problems). At baseline, $90.6 \%$ of the study sample met criteria for FA, with a mean number of FA symptoms equal to $6.1(\mathrm{SD}=1.2)$.

\section{Treatment effectiveness and changes in FA diagnosis}

The number of dropouts during treatment was low $n=11$ (16.7\%). No statistical differences were found between completers and dropouts in baseline measures of the number of binges $(p=.559)$ and purges $(p=.276)$, total number of FA symptoms $(p=.879)$, EDI-2 scores and the level of psychological symptoms measured on the SCL$90 \mathrm{R}(p>.05$ for all scales).

Table 1 contains the distribution of the BN severity group at baseline and post-treatment for the patients who completed treatment $(n=55)$. At baseline, moderate severity level had the highest level of prevalence (32.7\%), followed by extreme (25.5\%), severe $(23.6 \%)$ and mild (18.2\%) levels. After treatment, 14 patients were placed into the null severity group (the risk of obtaining abstinence from binging/purging episodes was $25.5 \%$; $95 \%$ CI: $15.8 \%$ to $38.3 \%$ ), $38.2 \%$ were placed in the mild severity group, $25.5 \%$ 
in the moderate, $9.1 \%$ in the severe and only $1.8 \%$ in the extreme group. The number of patients in the good responder group (change to a lower severity group after treatment) was 42 (risk=76.4\%; 95\%CI: $63.7 \%$ to $85.6 \%$ ).

Table 2 contains a comparison of FA severity between the good- and poor-responders groups. FA severity was statistically lower for patients in the good-responders group compared to patients in the poor responders group at the end of treatment. No statistically significant differences were found in patients' age of illness onset ( $p=.377$ ) or in the number of previous treatments for $\operatorname{ED~}(p=.100)$ when comparing these groups. However, good responders were older in age (means 30.7 versus 24.7 years-old; $p=.040$ ) and had a longer illness duration (means 12.7 versus 5.5 years; $p=.015$ ).

Table 3 compares changes in the presence of each FA symptom and in FA diagnosis between baseline and post-treatment for patients that completed treatment $(n=55)$. Only two FA symptoms did not obtain lower prevalence following the intervention (persistent desire or repeated unsuccessful attempts to quit and tolerance). The change in the prevalence of FA diagnosis was statistically significant $(p=.012)$, and decreased from $90.6 \%$ at baseline to $72.9 \%$ at post-treatment.

Table S1 (see Supplementary Information) contains pre-post mean comparisons for EDrelated measures, psychopathological symptom levels and the number of FA symptoms. Almost all these variables were significantly different when comparing baseline to posttreatment scores, with the exception of some EDI-2 scales (ineffectiveness, maturity fears, perfectionism, impulse regulation and social insecurity) and the SCL-90-R paranoid ideation score.

\section{Predictive capacity of baseline FA levels on treatment outcomes}

Table 4 contains three logistic regressions measuring the predictive capacity of the total number of FA symptoms registered at baseline on three treatment outcomes: being a "good responder" to treatment, total abstinence from binging/purging episodes and dropout. High FA severity predicted a lower likelihood of total abstinence from 
binging/purging episodes, though it did not predict risk of being a good responder to treatment nor dropout. Results were equivalent when including age and illness duration as covariates into the logistic models.

\section{Discussion}

The main aim of the present study, the first longitudinal study to assess FA in BN patients, was to ascertain whether FA severity at baseline predicted short-term treatment outcome. We also explored whether a brief psychoeducational intervention for $\mathrm{BN}$ was associated with reduced FA severity (measured by the number of reported FA symptoms) and FA diagnosis.

Our main finding is that, when comparing good-responders to treatment to poor responders, FA severity served as a predictor of short-term treatment outcome; patients with higher FA severity at baseline were less likely to obtain abstinence from bingeing/purging episodes after treatment. One potential interpretation of these findings is that FA is solely a marker of $\mathrm{BN}$ severity and that FA does not independently measure addictive mechanisms.

The second main finding is that FA severity and the prevalence of FA diagnosis was significantly reduced following a brief group psychoeducational intervention. In the current study, $90.6 \%$ of $\mathrm{BN}$ patients fulfilled criteria for FA diagnosis before starting treatment, and $72.9 \%$ of patients continued to meet the criteria for FA diagnosis following treatment. According to literature, a high percentage of patients with $\mathrm{BN}$ meet diagnostic criteria for FA, ranging from $81.5 \%$ (Granero et al., 2014) to 100\% (Meule et al., 2014). Meule and colleagues (Meule et al., 2014) found that the prevalence of FA was $70 \%$ higher in individuals with current $\mathrm{BN}$ relative to those with remitted $\mathrm{BN}$, although the cause of remission was not assessed (e.g., specific ED treatment, naturally occurring remission). 
Both, in clinical and general population samples, the most frequently endorsed FA symptom is "persistent desire or unsuccessful attempts to cut down" (Flint et al., 2014; Gearhardt et al., 2009, 2013; Meule et al., 2014; Pursey et al., 2016; Wolz et al., 2016). In our sample, this criterion, which denotes the feeling of lack of control, did not significantly change and remained the most prevalent FA symptom following treatment (95.8\%). This could be due to the fact that it is the only common symptom in BN and in FA. The criteria "continual use despite knowledge of adverse consequences" and "tolerance" have also shown high prevalence rates (Meule \& Gearhardt, 2014b) in ED samples. In this study, $84.4 \%$ of patients met the tolerance criterion at baseline and prevalence was not significantly reduced following treatment. One possible explanation for this lack of change could be that this symptom, indicating physiological dependence, needs more than the six weeks our treatment provided in order to notably improve.

The dropout rates obtained after this brief interventions were in line with previous literature (Agüera et al., 2013; Shapiro et al., 2007) and a total of 14 patients (27.3\%) were abstinent of binging/purging episodes during the final week of treatment. Although this reduction in $\mathrm{BN}$ symptomatology is significant, the prevalence of FA symptoms still remained relatively high. Future studies using longer treatment interventions should be carried out to determine whether these improvements are enduring.

Some limitations must be taken into account when interpreting the results of this study. First, the YFAS was not designed to detect short-term changes being that it explores people's behavior in the time frame of one year. However, we used this scale to measure changes after a brief intervention and requested our patients to complete the YFAS keeping in mind how they felt in relation to their eating behavior at that moment. A recent longitudinal study (over 18 months) with a non-clinical sample has shown that YFAS scores and FA diagnosis are relatively stable and therefore the YFAS could be a suitable tool to assess addictive-like eating behaviors over time and detecting treatment changes (Pursey et al., 2016). Second, we cannot speak of BN remission in our study, due to the fact that the time frame was too short and that patients had just initiated 
Food addiction and treatment response in $\mathrm{BN}$

treatment. However, these results are promising given that response to the initial stages of treatment predict treatment outcome (Agras et al., 2000; Brauhardt, De Zwaan, \& Hilbert, 2014; Fairburn, Agras, Walsh, Wilson, \& Stice, 2004).

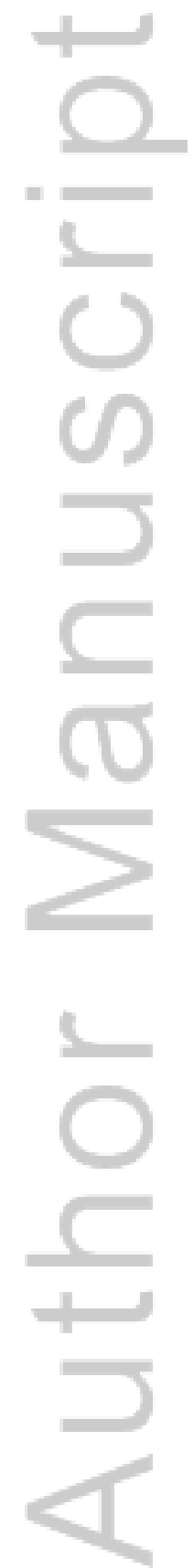

This article is protected by copyright. All rights reserved. 


\section{Conclusions}

Our results indicate that a brief psychological intervention is effective in reducing FA symptoms in BN patients. An important future direction would be to examine whether higher FA in BN patients is predictive of worse long-term treatment response and whether emphasizing mechanisms associated with addiction could be a useful adjunct to treatment in order to ensure long-term recovery in these patients.

\section{Conflict of interest.}

None.

\section{Acknowledgements}

This manuscript was supported by grants from Instituto de Salud Carlos III (FIS PI14/00290, CIBERobn, CIBERSAM) and co-funded by Fondos Europeos de Desarrollo Regional (FEDER). CIBERObn and CIBERSAM are both initiatives of ISCIII. This study was cofunded by FEDER funds/European Regional Development Fund (ERDF) - a way to build Europe and by a Ministerio de Economía y Competitividad grant (PSI2015-68701-R). The funders had no role in the study design, data collection and analysis, decision to publish, or preparation of the manuscript.

\section{References}

Agras, W. S., Crow, S. J., Halmi, K. A., Mitchell, J. E., Wilson, G. T., \& Kraemer, H. C. (2000). Outcome predictors for the cognitive behavior treatment of bulimia nervosa: data from a multisite study. The American Journal of Psychiatry, 157(8), 1302-8.

Agüera, Z., Riesco, N., Jiménez-Murcia, S., Islam, M. A., Granero, R., Vicente, E., ... Fernández-Aranda, F. (2013). Cognitive behaviour therapy response and dropout rate across purging and nonpurging bulimia nervosa and binge eating disorder: DSM-5 implications. BMC Psychiatry, 13, 285. http://doi.org/10.1186/1471-244X13-285 
APA. (2013). Diagnostic and Statistical Manual of Mental Disorders: DSM-5. Washington, DC: American Psychiatric Assocation.

Brauhardt, A., De Zwaan, M., \& Hilbert, A. (2014). The therapeutic process in psychological treatments for eating disorders: A systematic review. International Journal of Eating Disorders. http://doi.org/10.1002/eat.22287

Burmeister, J. M., Hinman, N., Koball, A., Hoffmann, D. A., \& Carels, R. A. (2013). Food addiction in adults seeking weight loss treatment. Implications for psychosocial health and weight loss. Appetite, 60(1), 103-110.

Clark, S. M., \& Saules, K. K. (2013). Validation of the Yale Food Addiction Scale among a weight-loss surgery population. Eat Behav, 14(2), 216-219. http://doi.org/10.1016/j.eatbeh.2013.01.002

Davis, R., Olmsted, M. P., \& Rockert, W. (1990). Brief group psychoeducation for bulimia nervosa: assessing the clinical significance of change. Journal of Consulting and Clinical Psychology, 58(6), 882-885. http://doi.org/10.1037/0022006X.58.6.882

Derogatis, L. R. (1990). SCL-90-R. Administration, scoring and procedures manual. Baltimore, MD: Clinical Psychometric Research.

Fairburn, C. G., Agras, W. S., Walsh, B. T., Wilson, G. T., \& Stice, E. (2004). Prediction of outcome in bulimia nervosa by early change in treatment. The American Journal of Psychiatry, 161(12), 2322-4. http://doi.org/10.1176/appi.ajp.161.12.2322

Fernández-Aranda, F., Alvarez-Moya, E. M., Martínez-Viana, C., Sánchez, I., Granero, R., Penelo, E., ... Peñas-Lledó, E. (2009). Predictors of early change in bulimia nervosa after a brief psychoeducational therapy. Appetite, 52(3), 805-8. http://doi.org/10.1016/j.appet.2009.03.013

Fernandez-Aranda, F., \& Turon, V. (1998). Trastornos de la alimentación. Barcelona: Masson.

Finner, H. (1993). On a Monotonicity Problem in Step-Down Multiple Test Procedures. Journal of the American Statistical Association, 88(423), 920-923. http://doi.org/10.1080/01621459.1993.10476358

Flint, A. J., Gearhardt, A. N., Corbin, W. R., Brownell, K. D., Field, A. E., \& Rimm, E. B. (2014). Food-addiction scale measurement in 2 cohorts of middle-aged and older women. Am J Clin Nutr, 99(3), 578-586. 
Garner, D. M. (1991). Eating Disorder Inventory-2. Odessa: Psychological Assessment Resources.

Garner, D. M. (1998). Inventario de Trastornos de la Conducta Alimentaria (EDI-2)Manual. Madrid: TEA.

Gearhardt, A. N., Boswell, R. G., \& White, M. A. (2014). The association of "food addiction" with disordered eating and body mass index. Eating Behaviors, 15(3), 427-33. http://doi.org/10.1016/j.eatbeh.2014.05.001

Gearhardt, A. N., Corbin, W., \& Brownell, K. (2009). Preliminary validation of the - Yale Food Addiction Scale. Appetite, 52(2), 430-436.

Gearhardt, A. N., White, M., Masheb, R., \& Grilo, C. (2013). An examination of food addiction in a racially diverse sample of obese patients with binge eating disorder in primary care settings. Compr Psychiatry, 54(5), 500-505.

Gearhardt, A. N., White, M., Masheb, R., Morgan, P., Crosby, R., \& Grilo, C. (2012). An examination of the food addiction construct in obese patients with binge eating disorder. Int J Eat Disord, 45(5), 657-663.

Granero, R., Hilker, I., Agüera, Z., Jiménez-Murcia, S., Sauchelli, S., Islam, M. a., ... Fernández-Aranda, F. (2014). Food Addiction in a Spanish Sample of Eating Disorders: DSM-5 Diagnostic Subtype Differentiation and Validation Data. European Eating Disorders Review, 22, 389-396. http://doi.org/10.1002/erv.2311

Hebebrand, J., Albayrak, Ö., Adan, R., Antel, J., Dieguez, C., de Jong, J., ... Dickson, S. L. (2014). "Eating addiction", rather than "food addiction", better captures addictive-like eating behavior. Neuroscience \& Biobehavioral Reviews, 47, 295306. http://doi.org/10.1016/j.neubiorev.2014.08.016

Ignacio, J., \& Sánchez, R. (2002). SCL-90-R: aplicación y análisis de sus propiedades psicométricas en una muestra de sujetos clínicos españoles. Psicopatología Clínica Legal Y Forense, 2(1), 5-19.

Lent, M. R., Eichen, D. M., Goldbacher, E., Wadden, T. A., \& Foster, G. D. (2014). Relationship of food addiction to weight loss and attrition during obesity treatment. Obesity (Silver Spring, Md.), 22(1), 52-5. http://doi.org/10.1002/oby.20512

Martínez-Azumendi, O., Fernández-Gómez, C., \& Beitia-Fernández, M. (2001). [Factorial variance of the SCL-90-R in a Spanish out-patient psychiatric sample]. Actas Españolas de Psiquiatría, 29(2), 95-102. Retrieved from http://www.ncbi.nlm.nih.gov/pubmed/11333527 
Meule, A., \& Gearhardt, A. N. (2014a). Five years of the Yale Food Addiction Scale: Taking stock and moving forward. Current Addiction Reports, 1(3), 193-205. http://doi.org/10.1007/s40429-014-0021-z

Meule, A., \& Gearhardt, A. N. (2014b). Food addiction in the light of DSM-5. Nutrients, 6(9), 3653-71. http://doi.org/10.3390/nu6093653

Meule, A., Hermann, T., \& Kübler, A. (2015). Food addiction in overweight and obese adolescents seeking weight-loss treatment. European Eating Disorders Review, 23(3), 193-8. http://doi.org/10.1002/erv.2355

Meule, A., von Rezori, V., \& Blechert, J. (2014). Food addiction and bulimia nervosa. European Eating Disorders Review, 22, 331-337. http://doi.org/10.1002/erv.2306

Pepino, M. Y., Stein, R. I., Eagon, J. C., \& Klein, S. (2014). Bariatric surgery-induced weight loss causes remission of food addiction in extreme obesity. Obesity (Silver Spring, Md.), 22(8), 1792-8. http://doi.org/10.1002/oby.20797

Pursey, K. M., Collins, C. E., Stanwell, P., \& Burrows, T. L. (2016). The stability of "food addiction" as assessed by the Yale Food Addiction Scale in a non-clinical population over 18-months. Appetite, 96, 533-8. http://doi.org/10.1016/j.appet.2015.10.015

Pursey, K. M., Stanwell, P., Gearhardt, A. N., Collins, C. E., \& Burrows, T. L. (2014). The prevalence of food addiction as assessed by the Yale Food Addiction Scale: a systematic review. Nutrients, 6(10), 4552-90. http://doi.org/10.3390/nu6104552

Shapiro, J. R., Berkman, N. D., Brownley, K. A., Sedway, J. A., Lohr, K. N., \& Bulik, C. M. (2007). Bulimia nervosa treatment: a systematic review of randomized controlled trials. The International Journal of Eating Disorders, 40(4), 321-36. http://doi.org/10.1002/eat.20372

Wilson, G. T., Fairburn, C. G., \& Agras, W. S. (1997). Cognitive-behavioral therapy for bulimia nervosa. In D. M. Garner \& P. E. Garfinkel (Eds.), Handbook of treatment for eating disorders (pp. 67-93). New York: Guilford.

Wolz, I., Hilker, I., Granero, R., Jiménez-Murcia, S., Gearhardt, A. N., Dieguez, C., .. Fernandez-Aranda, F. (2016). "Food addiction" in patients with eating disorders is associated with negative urgency and difficulties to focus on long-term goals. Frontiers in Psychology, 7. http://doi.org/10.3389/fpsyg.2016.00061 
Table 1

Distribution of the BN severity groups at baseline and post-treatment

\begin{tabular}{|c|c|c|c|c|c|c|}
\hline \multirow[b]{2}{*}{ Post-treatment } & \multicolumn{4}{|c|}{ Pre-treatment } & \multirow{2}{*}{\multicolumn{2}{|c|}{ Total }} \\
\hline & $\begin{array}{c}\text { Mild } \\
\text { (1-3 ep.) }\end{array}$ & $\begin{array}{c}\text { Moderate } \\
\text { (4-7 ep.) }\end{array}$ & $\begin{array}{l}\text { Severe } \\
\text { (8-13 ep.) }\end{array}$ & $\begin{array}{l}\text { Extreme } \\
\text { (>=14 ep.) }\end{array}$ & & \\
\hline Null (0 episodes) & 6 & 4 & 3 & 1 & 14 & $25.5 \%$ \\
\hline Mild (1-3 episodes) & 4 & 8 & 6 & 3 & 21 & $38.2 \%$ \\
\hline Moderate (4-7 episodes) & 0 & 6 & 2 & 6 & 14 & $25.5 \%$ \\
\hline Severe (8-13 episodes) & 0 & 0 & 2 & 3 & 5 & $9.1 \%$ \\
\hline Extreme (14-high) & 0 & 0 & 0 & 1 & 1 & $1.8 \%$ \\
\hline$=2$ & 10 & 18 & 13 & 14 & 55 & \\
\hline (utal & $18.2 \%$ & $32.7 \%$ & $23.6 \%$ & $25.5 \%$ & & \\
\hline
\end{tabular}

Note. Bold: good responder (change to a lower severity group at post-treatment). 
Table 2

Food addiction and treatment response in $\mathrm{BN}$

Comparison of patients with good response to treatment to patients with poor response to treatment (at baseline and following the intervention)

\begin{tabular}{|c|c|c|c|c|c|c|c|c|c|c|c|}
\hline & \multicolumn{2}{|c|}{$\begin{array}{l}\text { Good responders } \\
\qquad(n=42 ; 76.4 \%)\end{array}$} & \multicolumn{2}{|c|}{$\begin{array}{l}\text { Bad responders } \\
(n=13 ; 23.6 \%)\end{array}$} & \multicolumn{7}{|c|}{ Group comparison } \\
\hline & Mean & SD & Mean & SD & MD & SE & $t_{d f=49}$ & $p$ & $95 \%$ & $\mathrm{MD}$ & $|d|$ \\
\hline Number of FA criteria at baseline & 6.02 & 1.27 & 6.58 & 0.51 & 0.56 & 0.379 & 1.48 & .146 & -0.20 & 1.32 & 0.48 \\
\hline Number of FA criteria at post-treatment & 4.78 & 2.04 & 6.09 & 1.04 & 1.31 & 0.460 & 2.84 & $.008^{*}$ & 0.37 & 2.24 & $0.81^{\top}$ \\
\hline
\end{tabular}

Note. SD: standard deviation. Good-responders: change to a lower severity group after treatment.

*Bold: significant pre-post change. ${ }^{\dagger}$ Bold: moderate $(|d|>0.50)$ to high effect size $(|d|>0.80)$.

This article is protected by copyright. All rights reserved. 


\section{Table 3}

Changes in FA severity and diagnosis pre-and post-treatment

\begin{tabular}{|c|c|c|c|c|}
\hline & \multicolumn{2}{|c|}{ Prevalence (\%) } & \multirow[b]{2}{*}{$p$} & \multirow[b]{2}{*}{$|d|$} \\
\hline & Pre-treatment & Post-treatment & & \\
\hline Substance taken for longer period than intended & $81.3 \%$ & $60.4 \%$ & $.021^{*}$ & $0.50^{\top}$ \\
\hline Persistent desire or repeated unsuccessful attempts to quit & $100.0 \%$ & $95.8 \%$ & .500 & 0.29 \\
\hline Much time/activity to obtain, use, recover & $93.8 \%$ & $75.0 \%$ & $.006^{*}$ & $0.53^{\top}$ \\
\hline Important social-occupational-recreational activities reduced & $92.2 \%$ & $77.1 \%$ & $.039 *$ & 0.43 \\
\hline Use continues despite knowledge of adverse consequences & $73.4 \%$ & $50.0 \%$ & $.006^{*}$ & $0.50^{\top}$ \\
\hline Tolerance & $84.4 \%$ & $81.3 \%$ & .999 & 0.08 \\
\hline Characteristic withdrawal; substance taken to relieve withdrawal & $89.1 \%$ & $68.8 \%$ & $.013^{*}$ & $0.51^{\dagger}$ \\
\hline FA: positive diagnosis & $90.6 \%$ & $72.9 \%$ & $.012^{*}$ & $0.50^{\top}$ \\
\hline
\end{tabular}

Note. Exact McNemar test *Bold: significant pre-post change. ${ }^{\dagger}$ Bold: moderate $(|d|>0.50)$ to high effect size $(|d|>0.80)$. $(n=55)$. 
Table 4

Food addiction and treatment response in $\mathrm{BN}$

Predictive capacity of the number of FA symptoms at baseline on main treatment outcomes

\begin{tabular}{|c|c|c|c|c|c|c|c|c|c|}
\hline Criteria & B & S.E. & Wald & $p$ & OR & \multicolumn{2}{|c|}{$95 \% \mathrm{Cl}(\mathrm{OR})$} & $\mathrm{R}^{2}$ & AUC \\
\hline Being into the good responder group & -0.619 & 0.440 & 1.98 & .159 & 0.54 & 0.23 & 1.28 & .077 & .604 \\
\hline Abstinent binges/vomits & -0.622 & 0.28 & 4.85 & .018 & 0.54 & 0.31 & 0.93 & .146 & .682 \\
\hline Dropout from treatment & -0.043 & 0.276 & 0.02 & .877 & 0.96 & 0.56 & 1.65 & .001 & .500 \\
\hline
\end{tabular}

Note. $\mathrm{R}^{2}$ : Nagelkerke's-R ${ }^{2}$ coefficient. AUC: Area under the ROC curve. Bold: significant predictive capacity (.05 level).

Good-responders: change to a lower severity group after treatment. $(n=55)$.

This article is protected by copyright. All rights reserved. 